\title{
Numerical Analysis of Base Flowfield for a Four-Engine Clustered Nozzle Configuration T.-S. Wang
}

Reprinted from

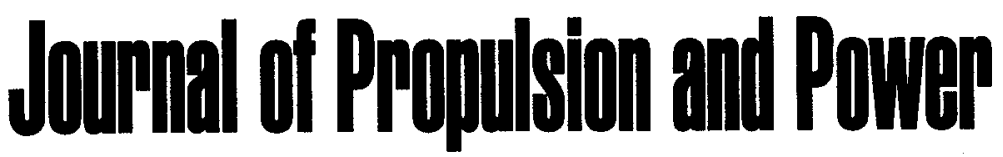

Volume 11, Number 5, Pages 1076-1078

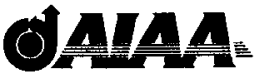

\section{A publication of the}

American Institute of Aeronautics and Astronautics, Inc.

370 L'Enfant Promenade, SW

Washington, DC 20024-2518 


\section{Numerical Analysis of Base Flowfield for a Four-Engine Clustered Nozzle Configuration}

\author{
Ten-See Wang* \\ NASA Marshall Space Flight Center, \\ Huntsville, Alabama 35812
}

\section{Introduction}

$\mathbf{E}$ XCESSIVE base heating has been a problem for many launch vehicles. For certain designs such as the direct dump of turbine exhaust inside and at the lip of the nozzle, the potential burning of the turbine exhaust in the base region can be of great concern. Accurate prediction of the base environment at altitudes is therefore very important during the vehicle design phase. Otherwise, undesirable consequences may occur.

In this study, the turbulent base flowfield of a cold flow experimental investigation ${ }^{1}$ for a four-engine clustered nozzle was numerically benchmarked using a pressure-based computational fluid dynamics (CFD) method. This is a necessary step before the benchmarking of hot flow and combustion flow tests can be considered. Since the medium was unheated air, reasonable prediction of the base pressure distribution at high altitude was the main goal. Several physical phenomena pertaining to the multiengine clustered nozzle base flow physics were deduced from the analysis.

\section{Numerical Modeling}

The basic equations employed in this study to describe the base flowfield for a four-engine clustered nozzle are the threedimensional, general-coordinate transport equations. These are equations of continuity, momentum, enthalpy, turbulent kinetic energy, and turbulent kinetic energy dissipation rate. A standard two-equation turbulence model is used to describe the turbulence.

To solve the system of nonlinear partial differential equations (PDEs), it uses finite difference approximations to establish a system of linearized algebraic equations. An adaptive upwind scheme was employed to approximate the convective terms of the momentum, energy, and continuity equations; the scheme is based on second- and fourth-order central differencing with artificial dissipation. The dissipation terms are constructed such that a fourth-order central and fourth-order damping scheme is activated in smooth regions, and a second-

Presented as Paper 93-1923 at the AIAA/SAE/ASME/ASEE 29th Joint Propulsion Conference and Exhibit, Monterey, CA, June 2830, 1993; received Aug. 6, 1993; revision received Oct. 11, 1994; accepted for publication Nov. 22, 1994. Copyright (C) 1995 by the American Institute of Aeronautics and Astronautics, Inc. No copyright is asserted in the United States under Title 17, U.S. Code. The U.S. Government has a royalty-free license to exercise all rights under the copyright claimed herein for Governmental purposes. All other rights are reserved by the copyright owner.

*Researcher, Computational Fluid Dynamics Branch. Member AIAA. order central and second-order damping scheme is used near shock waves. Viscous fluxes and source terms are discretized using second-order central difference approximation. A pressure-based predictor plus multicorrector solution method is employed so that flow over a wide speed range can be analyzed. The basic idea of this pressure-based method is to perform corrections for the pressure and velocity fields by solving a pressure correction equation so that velocity/pressure coupling is enforced, based on the continuity constraint at the end of each iteration. Details of the present numerical methodology are given in Ref. 2.

\section{Computational Grid Generation}

A typical layout of the computational grid is shown in Fig. 1. The four nozzles, which are conical with a cylindrical external shell, are equally spaced on a circular base. ${ }^{1}$ Due to the symmetrical nature of the flowfield, only one-eighth of this layout is generated and used for the actual calculation. The two sides of the pie-shaped grid, as shown in Fig. 1, are the symmetry planes. Two grid zones were created. The first zone started at the base and included the nozzle and the plume region. The second zone (the outer shell) comprises the ambient air and a portion of the expanded plume.

Three algebraic grids were generated for the purpose of this report. The difference among these three grids can be visualized by taking a section from the nozzle symmetry plane that lies in between the nozzle centerline and model centerline, as shown in Fig. 1. Grid A has 34,030 points, whereas the grid density for grid $B$ and $C$ is 113,202 points. An ambient-to-total-pressure ratio, $P_{a} / P_{0}=39 \times 10^{-4}$, is chosen as the nozzle operating condition. The grid lines near the nozzle lip of grid $\mathrm{C}$ are slanted to match the Prandtl-Meyer expansion.

\section{Boundary Conditions}

To start the calculation, an axisymmetric nozzle flow solution at the prescribed nozzle condition was carried out in a separate manner. The converged flow solution was then mapped to a three-dimensional nozzle flowfield. The nozzle lip, nozzle outer wall, and the base were specified as no-slip wall boundaries. The exit planes of zones 1 and 2 , the outer surface (shell) of zone 2 , and the inlet plane of zone 2 (flush with the base shield plane) were specified as exit boundaries. In addition, a fixed (ambient) pressure was imposed on the inlet plane of zone 2 in order to obtain a unique solution for the corresponding altitude. Flow properties at the wall, symmetry plane, and exit boundary were extrapolated from those of the interior domain. A tangency condition was applied at the symmetry planes.

\section{Results and Discussion}

At this ambient pressure, which corresponds to an altitude of $91,800 \mathrm{ft}$, the four exhaust plumes have interacted and a reverse jet is formed. The reverse jet impinges on the center of the base and spreads out, forming a wall jet. This wall jet may be choked once the ambient pressure is lower than a critical limit. A comparison of the computed radial base pressure profiles with data is shown in Fig. 2. In general, the peak pressure occurred at the base center and the base pressure decreased as the radial distance from the center of heat shield increased. 


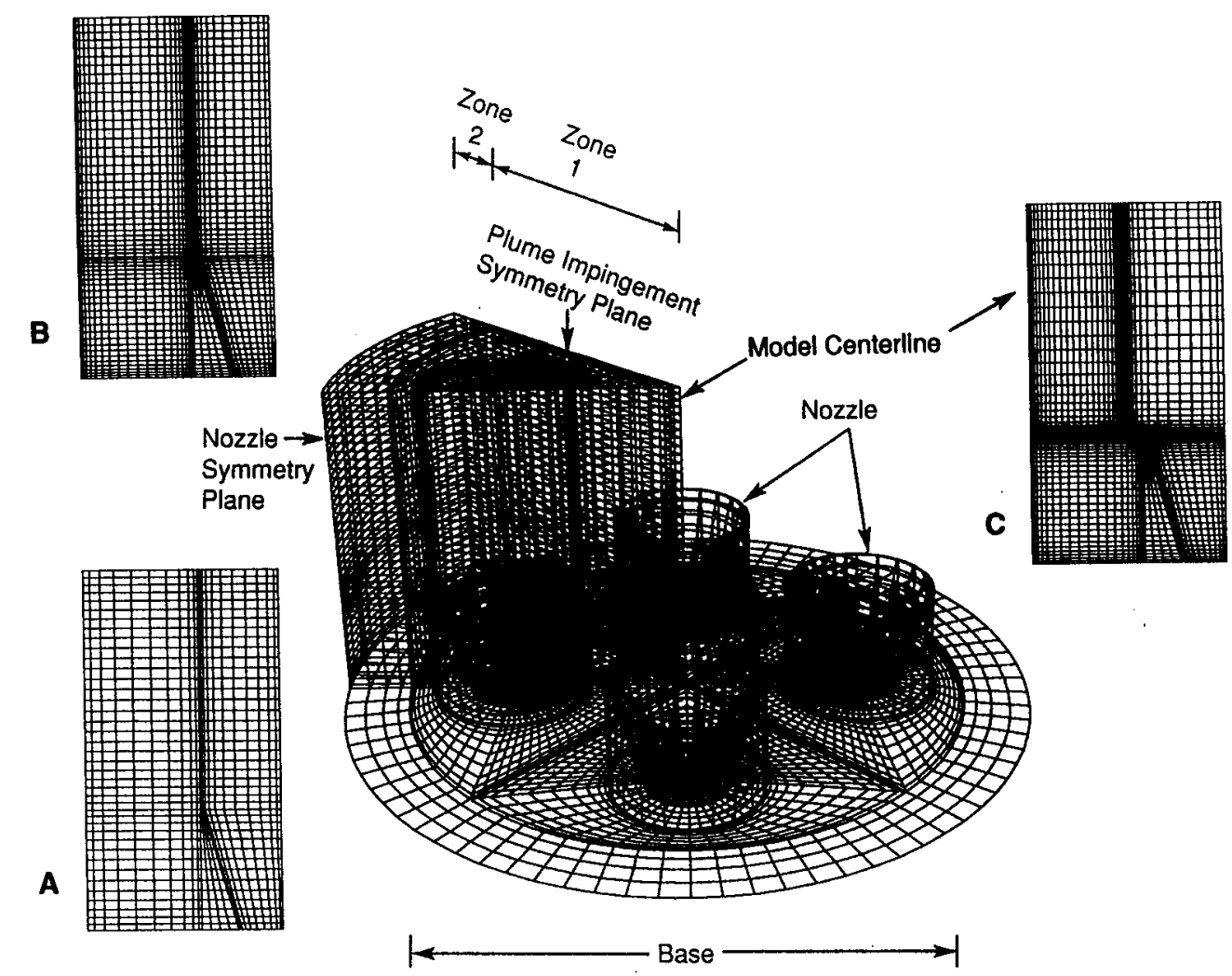

Fig. 1 Layout of a typical computational grid and slices of three different computational grids.

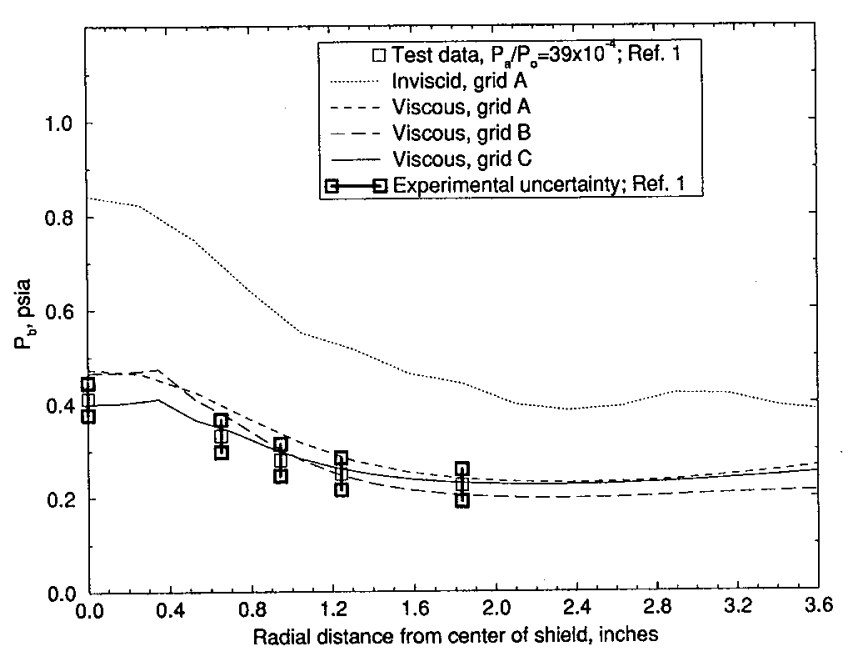

Fig. 2 Comparison of the radial base pressure distributions.

According to the inviscid multiengine clustered nozzle base flow theory proposed by Brewer, ${ }^{\prime}$ the predominant amount of the reverse flow originates from the detached shock portion of the "inviscid plume impingement line," rather than the attached oblique shock recompression portion; hence, the shear layer type of analysis ${ }^{3}$ for calculating the reverse flow is not applicable. The result of the inviscid flow solution on grid $A$ indicates that the lateral velocity at the plume boundary had inviscid flow reversal, emanating from behind the detached shock portion of the plume impingement. Albeit the absolute magnitudes were overpredicted, the predicted trend is encouraging and confirms Brewer's theory on the origination of the reverse jet. However, the overpredictions indicate the inviscid flow theory requires improvement and the vast improvement in viscous solution on grid $\mathrm{A}$ demonstrated just that. That is, the viscous effect determines the strength of the reverse jet by resolving the shear layer and the level

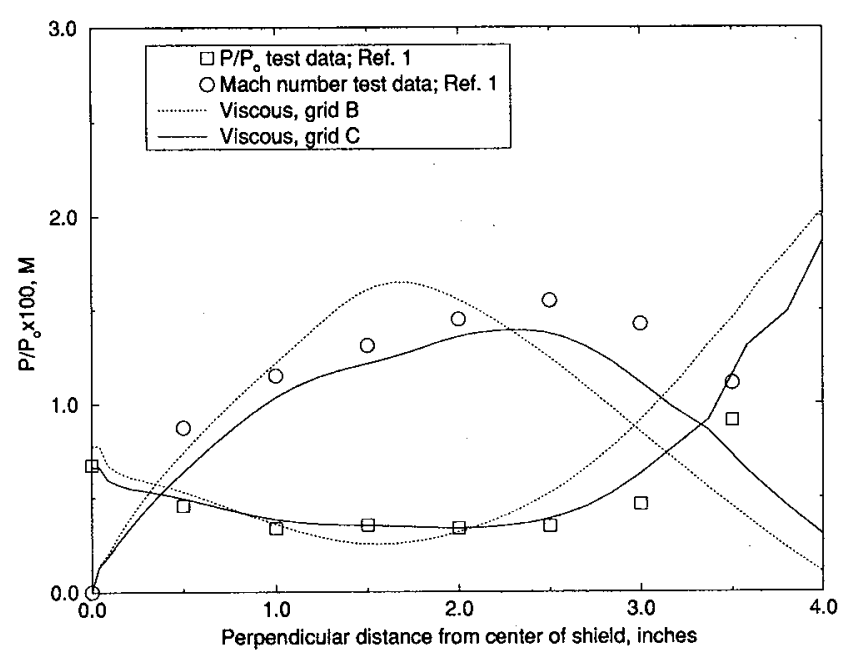

Fig. 3 Comparison of Mach number and static pressure variations along model centerline.

of the turbulence. The viscous grid B solution only improved slightly over that of viscous grid $A$, indicating the viscous resolution was not drastically improved with 3.3 times increase in grid density. Whereas the viscous solution of grid $\mathrm{C}$ matched the data very well, indicating the resolution of a PrandtlMeyer expansion is probably more efficient than the sheer increase of the grid density.

Mach number and static pressure comparisons along model centerline, as shown in Fig. 3, assess the goodness of the model prediction for the strength of the reverse jet. The grid B prediction that overpredicted the central base pressure previously, also peaked too early in Mach number profile and bottomed too early in static pressure profile, indicating the acceleration of the reverse jet was too close to the base. The grid $\mathrm{C}$ solution, on the other hand, followed the data reasonably well. This result illustrated the importance of both Prandtl-Meyer expansion and viscous effect. 


\section{Conclusions}

A three-dimensional, pressure-based CFD method is used to benchmark an experimental investigation of base flowfield for a four-engine clustered nozzle. The result of the analysis supported Brewer's inviscid flow theory ${ }^{1}$ that the lateral flow emanating from behind the detached shock portion of the plume impingement dominated the creation of the reverse jet. It also goes one step further by showing that the strength of the reverse jet is determined by the viscous effect. In addition, computationally efficient base flowfield solution is obtained through Prandtl-Meyer expansion resolved grid treatment.

\section{References}

'Brewer, E. B., and Craven, C. E., "Experimental Investigation of Base Flow Field at High Altitude for a Four-Engine Clustered Nozzle Configuration," NASA TND-5164, Feb. 1969.

'Wang, T.-S., and Chen, Y.-S., "Unified Navier-Stokes Flowfield and Performance Analysis of Liquid Rocket Engines," Journal of Propulsion and Power, Vol. 9, No. 5, 1993, pp. 678-685.

${ }^{3}$ Korst, H. H., Chow, W. L., and Zumwalt, G. W., "Research on Transonic and Supersonic Flow of a Real Fluid at Abrupt Increases in Cross Section," ME Tech. Rept. 392-5, Univ. of Illinois, Urbana, IL. 1959. 